Revista

\title{
Multi-Ensayos
}

Vol. 5, N 10

ISSN: 2412-3285

https://multiensayos.unan.edu.ni

DOI: https://doi.org/10.5377/multiensayos.v5i10.8883

\section{Experiencias, fortalezas y dificultades de la educación a distancia virtual}

\section{Experiences, strengths, and difficulties of virtual distance education}

Harold Alberto López Briones ${ }^{1}$

Recibido: 20 de mayo de 2019. Aceptado: 27 de junio de 2019

\section{RESUMEN}

En este ensayo se aborda las experiencias adquiridas, las fortalezas que desarrollaron los estudiantes del I año de Economía de la Facultad Regional Multidisciplinaria (FAREM-ESTELÍ), en relación al aprendizaje de la asignatura Informática Básica, la cual fue facilitada de manera virtual. Además se habla de las dificultades que fueron encontrándose durante este proceso de aprendizaje.

Palabras claves: experiencias; fortalezas; virtual; dificultades; aprendizajes.

\section{ABSTRACT}

This essay studies the lessons learned and strengths developed by the students of the first year of Economics of the Multidisciplinary Regional faculty (FAREM-ESTELÍ), in relation to the learning of the basic computer subject, which was facilitated in a virtual way. We also talk about the difficulties that were encountered during this learning process.

Keywords: experiences; strengths; virtual; difficulties; learning.

1 Docente UNAN-Managua/FAREM-Estelí. Correo electrónico: harlop79@hotmail.com.

(C) 2019 Revista Multi-Ensayos.

(c) (i) (-) Este trabajo está licenciado bajo una Licencia Internacional Creative Commons 4.0 Atribución-NoComercial-Compartirlgual. 


\title{
INTRODUCCIÓN
}

En la actualidad, el uso de tecnologías de la información y la comunicación (TIC'S) en los procesos educativos es indispensable, debido a que estas son utilizadas por los estudiantes y docentes para compartir información, comunicarse, e investigar cualquier tema de interés; es por esta razón que surge la necesidad en los docentes de utilizarlas para darle seguimiento a los aprendizajes que alcanzan los estudiantes, y también porque ofrecen diversidad de recursos de apoyo a la enseñanza (vídeos, wikis, foros, glosarios, cuestionarios) desarrollando creatividad, innovación, entornos de trabajo colaborativo y promoviendo el aprendizaje significativo en los estudiantes.

\begin{abstract}
"En líneas generales podríamos decir que las nuevas tecnologías de la información y comunicación son las que giran en torno a tres medios básicos: la informática, la microelectrónica y las telecomunicaciones; pero giran, no sólo de forma aislada, sino lo que es más significativo de manera interactiva e interconexionadas, lo que permite conseguir nuevas realidades comunicativas". (Cabero, 1998, pág. 198)
\end{abstract}

La Universidad Nacional Autónoma de Nicaragua (UNAN-Managua) propone en su modelo educativo que las TIC'S se utilizarán no solo para mejorar las prácticas pedagógicas en el aula, sino también para permitirles a los estudiantes otros escenarios de aprendizaje. (UNAN-MANAGUA, 2011, pág. 33).

En su crecimiento institucional la UNAN-Managua ha desarrollado programas de formación general (PFG), los cuales son coordinados por la dirección de educación a distancia virtual (DEDV). A partir el segundo semestre del 2017, la DEDV crea los cursos en línea de la asignatura Informática Básica, y en esta oportunidad la misma ha sido facilitada por mí persona a los estudiantes de primer año de Economía.

La educación virtual es una estrategia educativa que facilita el manejo de la información y que permite la aplicación de nuevos métodos pedagógicos enfocados al desarrollo de aprendizajes significativos, los cuales están centrados en el estudiante y en la participación activa. Permite superar la calidad de los recursos presenciales, se ajusta al horario personal de los estudiantes y facilita la interacción continua entre compañeros y el docente por medio virtual. (ecured, 2014)

Para el educador Jesús Lau, "la educación virtual está llenando espacios vacíos o áreas de profundidad que la educación presencial no ha podido realizar. Es un apoyo a la educación presencial y viceversa". (Lau, 2011)

El siguiente ensayo tiene como objetivos, describir las experiencias adquiridas, cómo se adquirieron estas experiencias, qué fortalezas desarrollaron, dónde aplicarán estas habilidades y qué deben mejorar los estudiantes de primer año de Economía de la Facultad Regional Multidisciplinaria, FAREM-Estelí, en relación al aprendizaje de la asignatura Informática Básica, facilitada de manera virtual. Además, describe las dificultades que surgieron durante este proceso de aprendizaje. 


\section{DESARROLLO}

Desde mi perspectiva, el docente virtual es facilitador del proceso enseñanza-aprendizaje, mediador, motivador, dinamizador y guía en las diferentes etapas del proceso de aprendizaje virtual. Un docente virtual debe poseer la capacidad de motivar, dinamizar los espacios comunitarios, valorar las contribuciones personales de los estudiantes, favorecer el trabajo en equipo y realizar un seguimiento personalizado de todos y cada uno de los estudiantes. También debe ajustarse al perfil de cada estudiante, porque cada quien impone su propio ritmo de aprendizaje.

Entre algunas de mis funciones como docente, puedo mencionar: 1) ofrecer orientación y asesoría al desarrollo de las actividades propuestas por el curso, además de evaluar todo el proceso a través de un seguimiento permanente, 2) facilitar el intercambio de experiencias e ideas, promoviendo el trabajo colaborativo para la comprensión personal y grupal del contenido del curso, y 3) brindar seguimiento y retroalimentación a las actividades de aprendizaje propuestas en el curso, ofreciendo sugerencias para la mejora continua de las asignaciones desarrollas por los estudiantes.

En el desarrollo de este curso, según los estudiantes de primer año de la carrera Economía aprendieron a: trabajar en línea haciendo uso de la plataforma virtual de la UNAN-Managua, conocer las conceptualizaciones básicas de la informática y la computadora, manejar nuevas técnicas de aprendizajes como vídeos y documentos digitales, utilizar de manera eficiente los programas de office como (Word, Excel, Power Point), crear carpetas y portafolios electrónicos, utilizar las normas APA para citar autores y buscar información en páginas web confiables.

En cuanto a cómo lo aprendieron, los estudiantes expresan que lo lograron a través de los laboratorios aplicados en clase, la lectura de documentos digitales, vídeos de cómo realizar las actividades propuestas, trabajos colaborativos, donde buscaban ayuda de algunos compañeros para la elaboración de las tareas asignadas en cada semana, así como consultas al docente en el laboratorio de clase y fuera de este, haciendo uso de foros para consultas creados en la plataforma virtual.

Los estudiantes comentan que desarrollaron las siguientes fortalezas: Manejo de forma eficaz y eficiente de los programas de office, habilidades de creatividad al realizar las tareas asignadas, resolución correcta de los ejercicios planteados en cada una de las unidades del curso, mejoramiento del hábito de lectura, comprensión y análisis de todas las tareas orientadas por el docente, elaboración de mapa conceptuales haciendo uso de herramientas virtuales como popplet.

También expresan que las habilidades adquiridas las aplicarán en su vida cotidiana, en el ámbito profesional y personal, ya que podrán desarrollar trabajos con una mejor calidad, así como ayudar a otras personas con el manejo del computador y de las herramientas que ofrece office en sus programas de aplicación.

Para alcanzar sus objetivos como estudiantes y futuros profesionales ellos exponen que deben mejorar en los siguientes aspectos: El hábito de lectura, ya que no están acostumbrados a leer documentos de 
manera digital, mejorar la velocidad para usar el teclado, la puntualidad para enviar los trabajos a la plataforma virtual, es decir, no dejarlos para la última hora, tener tolerancia y paciencia al momento de realizar los trabajos, dar buen uso a las herramientas informáticas y al computador.

También hubo algunas dificultades durante el seguimiento de este curso, entre las cuales destaco:

- Brecha digital, es decir, no todos los estudiantes tienen acceso a las herramientas tecnológicas para elaborar los trabajos.

- Fobia o temor por parte de algunos estudiantes al uso de tecnología en los procesos de aprendizajes.

- Algunos estudiantes argumentaron no tener acceso a internet, ya sea por no contar con la tecnología o recursos económicos para su uso.

- En ocasiones no se contaba con el acceso a internet en los laboratorios de computación, impidiendo la explicación del docente de cómo resolver las tareas asignadas en el curso.

- Incumplimiento de algunos estudiantes con el envío de trabajos asignados.

- Falta de práctica por los estudiantes.

- Debilidad en técnicas de lectura de algunos estudiantes.

- $\quad$ El tiempo de dedicación al aprendizaje virtual.

Algunas de estas dificultades pueden ser resueltas en un futuro, por ejemplo, mejorar los recursos tecnológicos con que cuenta la universidad, motivar a los docentes y estudiantes para que incorporen las tecnologías en los procesos educativos, así como crear cursos virtuales para otras asignaturas.

Además, la actividad del aprendizaje en un entorno virtual se centra en tres elementos: el primero es la calidad de los materiales didácticos y metodologías de trabajo sugeridas, el segundo la forma en que se van a realizar las evaluaciones y el seguimiento a estos estudiantes, y por último mejorar las relaciones entre docente y estudiantes, en cuanto a la comunicación por medios de tecnologías.

\section{CONCLUSIÓN}

Esta experiencia fue de gran importancia para los estudiantes, ya que ellos desarrollaron una serie de habilidades y capacidades que les permitirán ampliar horizontes en cuanto a lo profesional, así también crecieron en valores morales como la ética, la honestidad, la responsabilidad, y sobre todo el ser autodidactas, es decir, formar sus propios aprendizajes sin la necesidad de un docente.

Los cursos virtuales permiten a los estudiantes asumir compromisos personales en cuanto al cumplimiento de las tareas asignadas, enfocarse a las necesidades estudiantiles y laborales, y a formarse continuamente como profesionales.

El desarrollo de cursos virtuales permite a los estudiantes ser innovadores y creativos, ya que ante cierta situación presentada buscan nuevas alternativas de solución. 


\section{REFERENCIAS}

Cabero, J. (1998). Impacto de las nuevas tecnologías de la información y comunicación en las organizaciones. Granada-España: Grupo Editorial Universitario.

ecured. (11 de febrero de 2014). ecured.cu. Recuperado el 24 de noviembre de 2017, de ecured.cu: https:// www.ecured.cu/Educación_Virtual

Lau, J. (18 de Octubre de 2011). Educación a Distancia o Virtual. (S. M. González, Entrevistador) UNAN-MANAGUA. (2 de septiembre de 2011). Modelo Educativo. Managua, Nicaragua. 